\title{
FOREWORD: SPECIAL ISSUE OF JSC ON THE OCCASION OF MEGA 2019
}

\author{
ALESSANDRA BERNARDI, CARLOS D'ANDREA, AND THORSTEN THEOBALD
}

MEGA is the acronym for Effective Methods in Algebraic Geometry (and its equivalent in various languages). In this series of biennial international conferences, with the tradition dating back to 1990, MEGA 2019 was the 15th one and was held during June 17-21, 2019 in Madrid. The scope of effective methods in algebraic geometry is regarded in a broad sense, including their foundations, analysis, complexity, computational aspects, practical issues and applications. Through its particular format with a competitive submission process for the regular talks, the MEGA conference series facilitates to promote leading research in these fields.

The current special issue of the Journal of Symbolic Computation for MEGA 2019 features selected contributions related to the topics of the conference. Authors of accepted MEGA submissions were encouraged to submit their work, but the call was open to everyone. As such, the current outcome of the journal submission and refereeing process showcases current developments and research achievements in effective methods in algebraic geometry. The topics of the papers include algorithmic algebra, computational algebraic geometry, symbolic computation, commutative algebra, real geometry, algebraic number theory, convex algebraic geometry, as well as applications of these fields.

We hope that the reader enjoys the panorama of current research developments presented in the special issue. In the following short overview on the results, the papers are sorted in alphabetical order of the first author.

(1) In Autocovariance varieties of moving average random fields, Carlos Améndola and Viet Son Pham study the autocovariance functions of moving average random fields from an algebraic perspective. To do this, autocoinvariance varieties are defined, their dimension and degrees established, and their algebraic properties used to obtain statistical consequences such as identifiability of model parameters.

(2) Elena Angelini, Cristiano Bocci and Luca Chiantini in Catalecticant intersections and confinement of decompositions of forms introduce the new notion of confinement of decompositions for forms which allows to lower the number of parameters that one needs to consider, in order to find all the possible decompositions of a given set of data in terms of rank decompositions. They provide a shorter proof of a result by London that 3 general plane cubics have 2 simultaneous Waring decompositions of rank 6 and they compute that 4 general plane quartics have 18 different decompositions of rank 10.

(3) Strict inclusions of high rank loci by Edoardo Ballico, Alessandra Bernardi and Emanuele Ventura studies the high rank loci of a projective variety, that is, the closures of the sets of points whose corresponding rank is higher than the generic 
one. The authors provide classes of constructions for which strict inclusion occurs for two neighboring high rank loci.

(4) Mauro Beltrametti, Alessandro Logar and Maria-Laura Torrente's paper Quartic monoid surfaces with maximum number of lines focuses on a more detailed description of this family of surfaces, which is known to have 31 lines contained in it. They prove that there is an open subset parametrizing all these surfaces up to a projectivity, and also study the action of $\operatorname{PGL}(4, K)$ (for a field $K$ of characteristic zero) on these surfaces.

(5) In the paper Saturations of subalgebras, SAGBI bases, and U-invariants, the authors Anna Maria Bigatti and Lorenzo Robbiano address the problem of saturating a subalgebra with respect to a given polynomial. They present an algorithm that computes a set of generators and then they focus their attention on the graded case. They also apply their technique to the problem of directly computing semiinvariants.

(6) Janko Böhm, Wolfram Decker, Santiago Laplagne and Gerhard Pfister, in the paper Computing integral bases via localization and Hensel lifting, present a new, parallelizable and outperforming algorithm for constructing the normalization of a plane curve. Typical applications are the computation of adjoint ideals, the computation of Riemann-Roch spaces and the parametrization of rational curves.

(7) Francisco Jesús Castro-Jiménez and Helena Cobo, in their contribution On certain polynomial systems involving Stirling numbers of second kind, solve a special type of linear systems whose coefficients are polynomials involving the Stirling numbers of second kind in multivariate polynomial rings. These systems arise in the computation of the global b-functions: parametric Bernstein-Sato polynomials associated with certain hypergeometric ideals in the Weyl algebra.

(8) In the paper Voronoi cells of varieties, Diego Cifuentes, Kristian Ranestad, Bernd Sturmfels and Madeleine Weinstein generalize Voronoi cells and Voronoi decompositions of point sets to Voronoi cells and Voronoi decompositions of real algebraic varieties. In this setting, the Voronoi cells are convex semialgebraic sets. The paper studies the computational algebraic geometry of the Voronoi cells, in particular the algebraic boundary.

(9) Timothy Duff, Nickolas Hein and Frank Sottile use approximate solutions of a polynomial square subsystem to study their feasibility as a solution of the whole system in Certification for polynomial systems via square subsystems. To certify their extension, different approaches are considered, namely liaison pruning, Newton-Okounkov bodies, and intersection theory.

(10) Elisa Gorla, Daniela Mueller and Christophe Petit study in their paper Stronger bounds on the cost of computing Gröbner bases for HFE systems the multivariate polynomial systems of the Hidden Field Equations cryptosystem. Improved bounds on the solving degree and the last fall degree are given.

(11) In the paper On a tropical version of the Jacobian conjecture, Dima Grigoriev and Danylo Radchenko provide two different criteria for a tropical map between $n$-dimensional real spaces to be an isomorphism. Both criteria involve the use 
of Jacobian matrices, and analogues of the Jacobian conjecture in the tropical context.

(12) In Initial steps in the classification of maximal mediated sets, Jacob Hartzer, Olivia Röhrig, Timo de Wolff and Oguzhan Yürük take initial steps in classifying MMS both from a theoretical and a practical point of view. They show that MMS of simplices are isomorphic if and only if the simplices generate the same lattice up to permutations, and fully characterize the MMS for all simplices of sufficiently small dimensions and maximal 1-norms.

(13) The main result in Gregor Kemper's Computing quotients by connected solvable groups is a constructive version of a recent paper by Popov, where it is shown that if a connected solvable group $G$ acts on an irreducible variety $X$ over an algebraically closed field $K$, then $X$ has a $G$-stable dense open subset $U$ that admits a geometric quotient $U \rightarrow Y$. The algorithm is Gröbner basis free. All it requires are arithmetic operations and zero recognition in $K[X]$.

(14) The paper Computing representation matrices for the Frobenius on cohomology groups by Momonari Kudo presents algorithms to compute representation matrices for the Frobenius maps on cohomology groups in rather general settings. The computational complexity of these algorithms is studied, and computational experiments in Magma are provided.

(15) Daniel Perrucci and Marie-Françoise Roy present in their paper A new general formula to compute the Cauchy index with subresultants on an interval connections between Cauchy indices and subresultant polynomials which lead to improvements in the computation of the latter ones via subresultants.

(16) In Measuring the local non-convexity of real algebraic curves, Miruna-Stefana Sorea introduces and studies a new combinatorial object called the Poincaré-Reeb tree, whose role is to encode the shape of a smooth compact connected component of a real algebraic plane curve. The main results obtained in this paper are: measuring the non-convexity via the Poincaré-Reeb tree and the study of nested level curves of a real bivariate polynomial near a strict local minimum.

(17) Justyna Szpond in Unexpected hypersurfaces with multiple fat points shows the existence of unexpected hypersurfaces with multiple fat points. The author proves that the dimension of the linear system of quadrics passing through a Fermat configuration in $\mathbb{P}^{5}$ having multiplicity 3 at a general point is higher than expected. The author conjectures that the example extends to quadric in $\mathbb{P}^{2 k+1}$ passing through the Fermat configuration and having one triple and $k-1$ general double points.

The special issue is rooted in the MEGA conference 2019 in Madrid, for which we acknowledge the outstanding work of the local organizing team headed by María Emilia Alonso García (Universidad de Complutense in Madrid) and Rafael Sendra (Universidad de Alcalá, co-chair). We also thank all the committee members and volunteers involved in the organization of the conference. The executive committee consisted of

- María Emilia Alonso García (Universidad Complutense de Madrid),

- Alessandra Bernardi (University of Trento), 
- Frederic Bihan (Université Savoie Mont Blanc, Chambéry),

- Carlos D’Andrea (Universidad de Barcelona)

- Sandra Di Rocco (KTH Stockholm),

- Thorsten Theobald (Goethe University Frankfurt, chair),

- Mauricio Velasco (Universidad de los Andes, Bogotá),

- Josephine Yu (Georgia Institute of Technology).

For the preparation of this volume, we received the help from many people, whom we would like to thank cordially. Particular thanks to the authors of the papers for their submissions and to the anonymous referees, who provided valuable advice to assess the submissions comprehensively. Finally, we are grateful to the editor-in-chief of the Journal of Symbolic Computation, Hoon Hong, for his long years' support of the MEGA conference series and his support during the preparation of this special issue.

\section{Alessandra Bernardi}

Department of Mathematics, University of Trento, 38123 Trento, Italy

E-mail address: alessandra.bernardi@unitn.it

Carlos D'Andrea

Departament de Matemàtiques i Informàtica, Universitat de Barcelona, 08007 Barcelona, Spain

E-mail address: cdandrea@ub.edu

THORSTEN THEOBALD

Institute of Mathematics, Goethe University, 60054 Frankfurt, Germany

E-mail address: theobald@math.uni-frankfurt.de 